\title{
COMPARAÇÃO DO PERFIL EPIDEMIOLÓGICO DAS HEPATITES B E C EM UM SERVIÇO PÚBLICO DE SÃO PAULO
}

\author{
Camila Rodrigues Bressane CRUZ¹, Miriam Matsura SHIRASSU² e Wellington P. MARTINS ${ }^{3}$
}

RESUMO - Contexto - As hepatites virais constituem doença de notificação compulsória e apresentam grande importância em nosso meio devido à elevada prevalência. Objetivo - Comparar o perfil epidemiológico dos casos confirmados de hepatites B e C notificados no Núcleo de Vigilância Epidemiológica do Hospital do Servidor Público Estadual de São Paulo, São Paulo, SP, no período entre janeiro de 2004 e abril de 2007. Material - Os resultados foram analisados através do teste do qui ao quadrado. Resultados Observou-se 112 casos confirmados de hepatite B e 651 de hepatite C. Houve predominância do sexo masculino dentre os casos de hepatite $\mathrm{B}$ e do sexo feminino entre os de hepatite $\mathrm{C}$, com significância estatística. A faixa etária mais frequente foi de 30 a 39 anos, considerando-se hepatite $\mathrm{B}(P<0,01)$ e 40 a 49 anos para hepatite $\mathrm{C}(P=0,49)$. A astenia foi o sintoma mais relatado. Os fatores de risco predominantes foram: doenças sexualmente transmissíveis (hepatite $\mathrm{B} ; P<0,01$ ), tratamento cirúrgico e transfusão de sangue (hepatite $\mathrm{C} ; P<0,01)$. A confirmação por critério laboratorial foi predominante e quanto à forma clínica, observou-se evolução para hepatite crônica em $54,5 \%$ e $81,7 \%$ dos casos, respectivamente para hepatite $\mathrm{B}$ e $\mathrm{C}(P<0,01)$. A transmissão do vírus $\mathrm{B}$ ocorreu predominantemente por via sexual $(P<0,01)$, enquanto o vírus $\mathrm{C}$ foi predominantemente transmitido por transfusão $(P<0,01)$. Conclusões - Os dados referentes ao perfil epidemiológico dos pacientes foram semelhantes aos disponíveis na literatura nacional e internacional. Ressalta-se a importância da vigilância epidemiológica na determinação do perfil das hepatites virais, permitindo que se estabeleçam propostas adequadas de prevenção e rastreamento.

DESCRITORES: Hepatite B, epidemiologia. Hepatite C, epidemiologia. Vigilância epidemiológica. Notificação de doenças.

\section{INTRODUÇÃO}

As hepatites virais são doenças infecciosas provocadas por diferentes agentes etiológicos que apresentam características epidemiológicas, clínicas e laboratoriais distintas. A distribuição das hepatites virais é universal com variações de acordo com os agentes determinantes, sendo os principais os vírus $\mathrm{A}, \mathrm{B}, \mathrm{C}, \mathrm{D}$ e $\mathrm{E}$, tendo o homem como o único reservatório importante. Os vírus VHA e VHE têm transmissão fecal-oral, enquanto os vírus VHB, VHC, VHD são transmitidos mais frequentemente por via sexual, parenteral, percutânea e vertical. O período de incubação pode variar de 15 a 180 dias, de acordo com o tipo de vírus. As hepatites virais passaram a ser doença de notificação compulsória (DNC) a partir de 8 de dezembro de 2003, quando foi publicada pelo Ministério da Saúde do Brasil a Portaria no 2325 que definiu a relação de DNCs para o território nacional.

A grande importância das hepatites virais em saúde pública vem da sua grande prevalência e incidência e pela possibilidade de complicações das formas agudas e crônicas ${ }^{(4)}$. Bases de dados obtidos de bancos de sangue na América Latina revelaram que os portadores de VHB excedem os 6 milhões, estando o Brasil e outros países da América do Sul (como Colômbia, Venezuela e Peru) dentre aqueles que apresentam territórios de alta endemicidade ${ }^{(16)}$. Estudos mostraram que a frequência da infecção pelo vírus da hepatite B (VHB) no Brasil varia de $0,5 \%$ a $1,1 \%$ no sul do país até $1,5 \%$ a $3,0 \%$ na região centro e noroeste, podendo alcançar até $15 \%$ na região amazônica, considerada de alta endemicidade ${ }^{(16)}$. A prevalência de $\mathrm{VHB}$ em nosso país aumenta da região sul em direção ao noroeste, com taxas de anti-HBc que variam de $61,5 \%$ no Acre, $10,2 \%$ em São Paulo e 5,5\% no Rio de Janeiro e Santa Catarina ${ }^{(8)}$. O Ministério da Saúde estima que, no Brasil, pelo menos 15\% da população já entrou em contato com VHB e que 1\% da população apresenta formas crônicas ${ }^{(4)}$. Não se sabe precisamente a prevalência nacional do vírus da hepatite $\mathrm{C}(\mathrm{VHC})$, porém, há relatos que sugerem que essa taxa varie de $1 \%$ a $2 \%$ da população em geral ${ }^{(7)}$. Estudo realizado na região sudeste mostrou prevalência de 1,42\% de portadores de anti-VHC na cidade de São Paulo. Dados provenientes do Centro de Vigilância Epidemiológica (CVE) revelam a confirmação de 3.649

Trabalho realizado no Núcleo de Vigilância Epidemiológica, Serviço de Medicina Social, Divisão Prevenir, Hospital do Servidor Público Estadual, São Paulo, SP.

${ }^{1}$ Núcleo de Vigilância Epidemiológica do Hospital do Servidor Público Estadual (HSPE); ${ }^{2}$ Divisão Prevenir do HSPE, São Paulo, SP; ${ }^{3}$ Hospital das Clínicas da Faculdade de Medicina de Ribeirão Preto da Universidade de São Paulo, Ribeirão Preto, SP.

Correspondência: Dra. Camila Rodrigues Bressane Cruz - Avenida Ibirapuera, 981 - 6º andar - sala 612 - 04029-000 - São Paulo, SP. E-mail: camila_bressane@hotmail.com 
casos de hepatite B e 6825 casos de hepatite $\mathrm{C}$ na cidade de São Paulo, no período de 1998 a 2006, dentre um total de 52.493 casos investigados em todo o Estado de São Paulo(5).

Os objetivos do presente estudo foram: analisar o perfil epidemiológico dos casos confirmados de hepatites pelos vírus B e C notificados no Hospital do Servidor Público Estadual (HSPE), São Paulo, SP, no período de janeiro de 2004 a abril de 2007 e comparar o perfil epidemiológico dos mesmos no HSPE com os dados disponíveis para o estado de São Paulo, bem como na literatura internacional.

\section{MÉTODOS}

Os dados analisados são provenientes das fichas de notificação de hepatite viral preenchidas no Núcleo de Vigilância Epidemiológica do Serviço de Medicina Social do HSPE no período de janeiro de 2004 a abril de 2007, quando foram investigados 826 casos de hepatite, tendo sido confirmados 780. Dentre eles, ocorreram 112 casos de hepatite B, 651 de hepatite C, 16 apresentaram hepatite B e $\mathrm{C}$ e 1 caso foi diagnosticado como hepatite A.

Foram considerados casos confirmados aqueles que preencheram as condições de caso suspeito: presença de icterícia, febre, mal estar geral, aumento de transaminases ou, quando assintomáticos, sofreram exposição a uma fonte de infecção bem documentada (hemodiálise, acidente ocupacional, comunicante de caso de hepatite, uso de drogas endovenosas, entre outros) e apresentaram marcadores sorológicos reagentes: $\mathrm{Hbs} \mathrm{Ag}, \mathrm{HbeAg}$ e anti-HBc IgM nos casos de hepatite B e anti-HCV e PCR positivo para HCV, nos casos de hepatite $\mathrm{C}$.

Foram analisados no presente estudo os casos confirmados de hepatite $\mathrm{B}$ e de hepatite $\mathrm{C}$ segundo as seguintes características: sexo, raça, escolaridade, faixa etária, fatores de risco, história de vacinação contra hepatite $\mathrm{B}$, história anterior de alcoolismo e icterícia, sinais e sintomas, critério diagnóstico, forma clínica, mecanismo provável de infecção e evolução. Os dados constantes nas fichas foram provenientes de investigação epidemiológica realizada nos prontuários de pacientes, declarações de óbito, resultados de exames laboratoriais e laudos anatomopatológicos disponíveis tanto nos prontuários quanto no sistema informatizado do HSPE. Os dados obtidos foram analisados aplicando-se o teste do qui ao quadrado $\left(\chi^{2}\right)$ a fim de comparar as proporções encontradas entre as hepatites do tipo B e C. Foi utilizado programa Epi Info ${ }^{\mathrm{TM}}$ (Centers for Disease Control and Prevention, Atlanta, GA, EUA) para a realização dos testes estatísticos.

\section{RESULTADOS}

Dentre os casos de hepatite B o sexo masculino foi predominante, correspondendo a $62,5 \%$ dos casos. Por outro lado, o sexo feminino predominou discretamente $(51,5 \%)$ dentre os casos de hepatite C. Houve significância estatística com relação ao sexo $(P<0,01)$. Com relação à raça, observou-se a seguinte distribuição respectivamente para hepatite B e C (excluindo-se os dados ignorados): 75,7\% e
$84 \%$ corresponderam à raça branca $(P=0,06), 7,3 \%$ e $8,5 \%$ corresponderam à raça negra $(P=0,71), 7,3 \%$ e $1,7 \%$ foram da raça amarela $(P<0,01)$ e $9,7 \%$ e $5,8 \%$ eram pardos $(P=0,18)$.

A distribuição segundo a faixa etária pode ser visualizada na Tabela 1. A comparação entre hepatite B e C para as diferentes faixas etárias revelou significância estatística na faixa etária de 30 a 39 anos $(P<0,01)$.

TABELA 1. Distribuição de casos confirmados de hepatite segundo faixa etária

\begin{tabular}{lccccc}
\hline & \multicolumn{2}{c}{ Hepatite B } & \multicolumn{2}{c}{ Hepatite C } \\
& $\mathbf{n}$ & $(\%)$ & $\mathbf{n}$ & $(\%)$ & $P$ \\
\hline$<20$ anos & 2 & 1,8 & 11 & 1,7 & 0,74 \\
20 a 29 anos & 8 & 7,1 & 26 & 4,0 & 0,21 \\
30 a 39 anos & 32 & 28,6 & 104 & 16,0 & $<0,01$ \\
40 a 49 anos & 28 & 25,0 & 183 & 28,1 & 0,49 \\
50 a 59 anos & 23 & 20,5 & 157 & 24,1 & 0,40 \\
60 a 69 anos & 14 & 12,5 & 103 & 15,8 & 0,36 \\
$>70$ anos & 5 & 4,5 & 67 & 10,3 & 0,05 \\
\hline Valor de $P$ obrido pelo reste do qui a quadrado & & & &
\end{tabular}

O campo escolaridade foi preenchido na maior parte das fichas epidemiológicas com o código 9 (ignorado), correspondendo a $39,3 \%$ dos casos de hepatite B e 51,5\% dos casos de hepatite $\mathrm{C}$. A faixa de escolaridade mais frequente foi acima de 12 anos (21,4\% para hepatite B e 18,3\% para hepatite C). Não houve diferença estatisticamente significante com relação à escolaridade.

Quanto à vacinação contra hepatite $\mathrm{B}$, apenas $4,5 \%$ dos pacientes diagnosticados com VHB realizaram o esquema completo, predominando aqueles que não foram vacinados $(67 \%)$. Dentre os casos de hepatite C, $11,1 \%$ realizaram vacinação completa e a maior parte $(50,8 \%)$ não foi vacinada contra hepatite B. A ausência da vacinação foi significantemente mais frequente dentre os casos de hepatite $\mathrm{B}(P<0,01)$.

Com relação à história anterior de icterícia, observou-se que foi positiva em 18,8\% dos casos de hepatite B e 8,6\% dos casos de hepatite $\mathrm{C}(P<0,01)$. Dentre os pacientes com hepatite $\mathrm{B}, 14,3 \%$ apresentaram antecedente de alcoolismo e naqueles com hepatite $\mathrm{C}$, essa taxa correspondeu a $21,3 \%$. Não houve significância estatística na comparação destes dados.

A Tabela 2 mostra a distribuição segundo exposição a fatores de risco. Observa-se que o antecedente de doenças sexualmente transmissíveis foi mais prevalente entre os casos de hepatite $\mathrm{B}(P<0,01)$; por outro lado, ocorreu maior prevalência de tratamento cirúrgico $(P<0,01)$ e transfusão de sangue $(P<0,01)$ entre os casos de hepatite $\mathrm{C}$. A análise da Figura 1 mostra que a astenia foi o sintoma mais frequente tanto entre os casos de hepatite B quanto de hepatite C.

$\mathrm{O}$ diagnóstico foi confirmado por critério clínicolaboratorial em $45,5 \%$ dos casos de hepatite B e 39,5\% dos casos de hepatite C. A confirmação laboratorial correspondeu a $54,5 \%$ e $60,8 \%$ dos casos, respectivamente. Esta diferença não foi estatisticamente significante. Não houve confirmação diagnóstica por critério clínico-epidemiológico.

Com relação à forma clínica no momento da notificação, observou-se que $54,5 \%$ dos casos de hepatite B e $81,7 \%$ dos casos de hepatite $\mathrm{C}$ apresentaram-se como hepatite crônica. As 
TABELA 2. Distribuição de casos confirmados de hepatite segundo exposição a fatores de risco

\begin{tabular}{|c|c|c|c|c|c|c|c|}
\hline \multirow[b]{2}{*}{ Fatores de risco } & \multicolumn{3}{|c|}{ Hepatite B (\%) } & \multicolumn{3}{|c|}{ Hepatite C (\%) } & \multirow[b]{2}{*}{$\mathbf{P}$} \\
\hline & Sim & Não & Ign. & Sim & Não & Ign. & \\
\hline $\begin{array}{l}\text { Transfusão de } \\
\text { sangue }\end{array}$ & 8,1 & 89,3 & 2,6 & $23,9 *$ & 67 & 9,1 & $<0,01$ \\
\hline Hemodiálise & $4,5 *$ & 92,8 & 2,7 & 2,3 & 89,2 & 8,5 & 0,01 \\
\hline Drogas injetáveis & 1,8 & 90,2 & 8 & $9,5 *$ & 76,1 & 14,4 & $<0,01$ \\
\hline Drogas inaláveis & 1,8 & 90,2 & 8 & $7,9 *$ & 76,6 & 15,5 & 0,02 \\
\hline $\begin{array}{l}\geq 3 \text { parceiros } \\
\text { sexuais/ano }\end{array}$ & $19,6^{*}$ & 45,6 & 34,8 & 9,2 & 48,3 & 42,5 & $<0,01$ \\
\hline DSTs & $22,3 *$ & 52,7 & 25 & 10,1 & 58,5 & 31,4 & $<0,01$ \\
\hline $\begin{array}{l}\text { Medicamentos } \\
\text { injetáveis }\end{array}$ & 16,1 & 66,1 & 17,8 & 19,3 & 56,5 & 24,2 & 0,22 \\
\hline $\begin{array}{l}\text { Exposição a } \\
\text { sangue/secreções }\end{array}$ & 5,4 & 84,8 & 9,8 & 9,7 & 73,9 & 16,4 & 0,09 \\
\hline $\begin{array}{l}\text { Tratamento } \\
\text { cirúrgico }\end{array}$ & 12,5 & 78,6 & 8,9 & $25,3 *$ & 59,9 & 14,8 & $<0,01$ \\
\hline $\begin{array}{l}\text { Tratamento } \\
\text { dentário }\end{array}$ & 0 & 15,2 & 84,8 & 5,2 & 51,5 & 43,3 & - \\
\hline Parto normal & 1,8 & 31,2 & 67 & 3,3 & 29,3 & 67,4 & - \\
\hline Acupuntura & 0 & 79,5 & 20,5 & 0,9 & 76,1 & 23 & - \\
\hline Tatuagem & 0 & 92,9 & 7,1 & 1,9 & 84,9 & 13,2 & - \\
\hline Piercing & 2,7 & 90,2 & 7,1 & 1,2 & 86,1 & 12,7 & 0,41 \\
\hline $\begin{array}{l}\text { Acidente } \\
\text { percutâneo }\end{array}$ & 0 & 95,5 & 4,5 & 1,4 & 85,2 & 13,4 & - \\
\hline Transplante & 0 & 78,6 & 21,4 & 1,4 & 84,6 & 14 & - \\
\hline
\end{tabular}

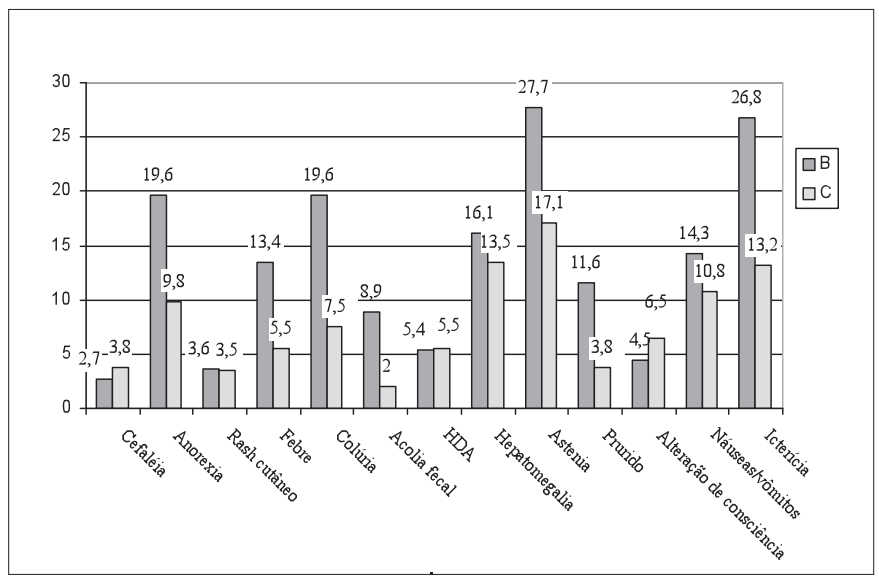

FIGURA 1. Distribuição de casos confirmados de hepatite segundo sinais e sintomas (percentagem)

formas agudas ocorreram em $11,6 \%$ e $0,6 \%$, respectivamente. Portadores assintomáticos corresponderam a 18,8\% e 1,2\% da amostra, enquanto a infecção assintomática ocorreu em $2,7 \%$ e $0,9 \%$, respectivamente para hepatite B e C. Dentre os casos de hepatite $\mathrm{B}, 12,5 \%$ tiveram a forma clínica registrada como ignorada; essa taxa correspondeu a $15,6 \%$ dos casos de hepatite C. A comparação entre hepatite B e C revelou significância estatística para as formas agudas, crônicas e portadores assintomáticos $(P<0,01)$. Quanto à evolução dos casos, por ocasião do encerramento da investigação epidemiológica, observou-se que a maior parte $(46,4 \%$ dos casos de hepatite $\mathrm{B}$ e $70,5 \%$ de hepatite $\mathrm{C}$ ) correspondeu à hepatite crônica $(P<0,01)$. Os óbitos corresponderam a apenas $7,1 \%$ e $4,5 \%$ dos casos, respectivamente $(P=0,27)$. A cura ocorreu em $9,8 \%$ dos casos de hepatite $\mathrm{B}$ e $0,6 \%$ para hepatite $\mathrm{C}(P<0,01)$. Os portadores crônicos corresponderam a $17,9 \%$ e $2 \%(P<0,01)$, enquanto os dados ignorados ocorreram em $18,8 \%$ e $22,4 \%$, respectivamente para hepatite B e C.

A Tabela 3 revela que, excluindo-se os dados ignorados, a transmissão sexual foi a mais frequente entre os casos de hepatite $\mathrm{B}$, enquanto a transfusão de hemoderivados predominou entre os casos de hepatite $\mathrm{C}$. A análise estatística mostrou que a transmissão sexual foi significativamente mais frequente para os casos de hepatite $\mathrm{B}(P<0,01)$; que a via transfusional foi significativamente mais frequente para hepatite $\mathrm{C}(P<0,01)$ e que outros mecanismos de transmissão (inclusive drogas injetáveis, transmissão vertical, tratamento cirúrgico e dentário, domiciliar e acidente de trabalho) foram mais frequentes para hepatite $\mathrm{C}(P<0,01)$. Deve-se considerar, entretanto, a grande proporção de dados ignorados: $61,6 \%$ para hepatite B e $64,5 \%$ para hepatite $\mathrm{C}$.

TABELA 3. Distribuição de casos confirmados de hepatite B e C quanto à provável fonte/mecanismo de infecção (exceto ignorado)

\begin{tabular}{lccccc}
\hline & \multicolumn{2}{c}{ Hepatite B } & \multicolumn{2}{c}{ Hepatite C } \\
Mecanismo/fonte & $\mathbf{n}$ & $\%$ & $\mathbf{n}$ & $\%$ & $\boldsymbol{P}$ \\
\hline Sexual & 36 & 32,1 & 60 & 9,2 & $<0,01$ \\
Transfusão & 4 & 3,6 & 97 & 14,9 & $<0,01$ \\
Outros & 3 & 2,7 & 75 & 11,5 & $<0,01$ \\
\hline
\end{tabular}

\section{DISCUSSÃO}

A análise dos dados confirmou, em grande parte, o perfil epidemiológico já previamente descrito na literatura nacional e internacional. Estudo do perfil epidemiológico de pacientes atendidos em Manaus no período de 1997 a $2001^{(3)}$ mostrou prevalência de $70,2 \%$ do sexo masculino dentre os casos de hepatite B e 53,5\% para hepatite C. A faixa etária predominante para hepatite B foi de 40 a 54 anos, enquanto para hepatite $\mathrm{C}$ observou-se a mesma prevalência para as faixas de 20 a 39 anos e maior que 54 anos $^{(3)}$, assemelhandose ao perfil do HSPE. Estudo epidemiológico realizado em quatro cidades brasileiras mostrou alta soroprevalência de anti-HBc na infância, com taxas crescentes a partir da faixa etária de 21 a 30 anos, além de predomínio estatisticamente significante no sexo masculino ${ }^{(15)}$. Tal perfil equipara-se ao da presente amostra, embora não haja ocorrido alta prevalência na infância, fato que decorre do perfil da população atendida no hospital, que é em sua maioria de pacientes idosos. Nos Estados Unidos, a faixa etária de 30 a 49 anos apresenta maior prevalência de hepatite $C$, enquanto os menores de 20 anos e maiores de 50 apresentam as menores taxas. Esse perfil é semelhante em países do norte da Europa e Austrália. Por outro lado, em países como Turquia, Espanha, Itália, Japão e China, a maior prevalência de infecção pelo VHC ocorre em indivíduos acima de 50 anos, demonstrando o efeito de coorte que sugere a ocorrência de maior risco de infecção 
pelo VHC de 40 a 60 anos atrás nesses países ${ }^{(2)}$. Com relação à etnia, na América Latina, a infecção pelo VHB tem sido mais frequente em brancos e pardos ${ }^{(16)}$. Nos Estados Unidos, por outro lado, as taxas de infecção pelo VHC são maiores em negros não-hispânicos do que em brancos não-hispânicos e mexicanos ${ }^{(13)}$.

Quanto ao mecanismo de infecção, nos Estados Unidos, $60 \%$ dos indivíduos infectados pelo VHC adquiriram a doença através do uso de drogas injetáveis ${ }^{(13)}$. Esse modo de transmissão também tem predominado nos últimos 40 anos na Austrália e países do norte, sul e oeste europeu ${ }^{(2,10,11)}$. A análise do perfil epidemiológico da hepatite $\mathrm{C}$ sugere que a transmissão de $\mathrm{HCV}$ em países desenvolvidos ocorre predominantemente por uso de drogas injetáveis, via transfusional e sexual, enquanto em países em desenvolvimento, as principais vias são: uso de medicamentos injetáveis e transfusões ${ }^{(2,17)}$. Estudo brasileiro que analisou 1.594 pacientes com anti-HCV positivo no período de 1975 a 2003, mostrou prevalência de $44,8 \%$ infectados por transfusão de hemoderivados, $4,5 \%$ por drogas endovenosas e $47 \%$ por via ignorada ${ }^{(7)}$. Outro estudo analisou o perfil de 100 doadores de sangue que apresentaram anti-HCV positivo na região do interior do Estado de São Paulo, mostrando que as vias de infecção foram: $26 \%$ por uso de drogas endovenosas ilícitas, $48 \%$ devido ao uso de drogas endovenosas (anabolizantes) e 15\% devido à transfusão de sangue $^{(14)}$. Em Manaus ${ }^{(3)}, 1,8 \%$ dos pacientes com hepatite B informaram uso de drogas injetáveis; tal taxa foi nula entre os pacientes com hepatite C. Este mesmo estudo mostrou prevalência de transfusão sanguínea de 30,3\% e 64,2\%, respectivamente para hepatite $\mathrm{B} \mathrm{e} \mathrm{C}^{(1)}$. Com relação à hepatite $\mathrm{B}$, a via sexual é considerada a mais frequente na América Latina $^{(15)}$, tendo sido também na presente amostra. Dados do sistema holandês de vigilância epidemiológica no período de 2002 a 2005 mostraram que a via sexual foi predominante principalmente nos casos de infecção aguda pelo vírus $\mathrm{B}^{(9)}$.

Estudo realizado na região sul do Brasil com 1.090 mulheres grávidas revelou prevalência de transmissão vertical de 5,56\% entre mães portadoras do $\mathrm{VHC}^{(12)}$. A taxa de transmissão vertical deste vírus pode variar de $4 \%$ a $7 \%$ por gestação e ocorre apenas quando o HCV RNA é detectável no soro materno na ocasião do parto ${ }^{(2)}$. Estudos internacionais identificaram taxas de transmissão vertical do VHC que variaram de 3\% a $37 \%$ de acordo com a viremia materna e a presença de coinfecção pelo HIV ${ }^{(18)}$. No norte da Itália, observou-se taxa de 4,2\% de transmissão após seguimento de HCV-RNA e anti-HCV em crianças de mães portadoras do VHC durante 18 meses $^{(18)}$. Com relação ao VHB, a transmissão vertical é muito frequente em países do leste asiático e da Oceania ${ }^{(1)}$. Na Holanda, no período de 2002 a 2005, observou-se que a transmissão vertical ocorreu em $40 \%$ dos casos de hepatite $\mathrm{B}$ crônica em regiões endêmicas ${ }^{(9)}$.

A Tabela 4 permite comparar as informações provenientes da presente amostra com dados disponíveis para o Estado e município de São Paulo no período de 1998 a 2006 com relação ao mecanismo provável de infecção ${ }^{(5)}$. Observa-se grande semelhança, inclusive pela alta proporção de dados ignorados. A tabela revela ainda a falta de informação com relação à hepatite $\mathrm{B}$, com taxas nulas que, provavelmente, não correspondem à realidade.

TABELA 4. Distribuição de casos confirmados de hepatite B e C segundo fonte/mecanismo provável de infecção no Estado de São Paulo*, município de São Paulo* e no HSPE

\begin{tabular}{lcccccc}
\hline & \multicolumn{2}{c}{ Estado (\%) } & \multicolumn{2}{c}{ Município SP(\%) } & \multicolumn{2}{c}{ HSPE (\%) } \\
Fonte/mecanismo & Hep. B & Hep. C & Hep. B & Hep. C & Hep. B & Hep. C \\
\hline Sexual & 26 & 10 & 36,9 & 11,2 & 32,1 & 9,2 \\
Transfusional & 3 & 16 & 2,7 & 15,4 & 3,6 & 14,8 \\
Drogas injetáveis & 3 & 21 & 2,6 & 17,8 & 0 & 8,3 \\
Vertical & 2 & 0 & 1,1 & 0,5 & 0 & 0,3 \\
Acidente de trabalho & 0 & 1 & 0,4 & 0,6 & 0 & 0,6 \\
Domiciliar & 3 & 0 & 2,6 & 1,1 & 0 & 0,2 \\
Trat. cirúrgico/dentário & 3 & 4 & 2,7 & 6,2 & 0 & 0,8 \\
Outro & 4 & 8 & 2,1 & 5,1 & 2,7 & 1,4 \\
Branco/ignorado & 56 & 40 & 48,9 & 42,1 & 61,6 & 64,4 \\
\hline$*$ = dados obtidos de: Ferreira C. Silveira T. & & & & &
\end{tabular}

Quanto à forma clínica, a literatura mostra que a prevalência de formas crônicas (inclusive cirrose hepática) em Manaus $^{(3)}$ foi de $81,5 \%$ para hepatite B e 70,5\% para hepatite $\mathrm{C}$, enquanto os portadores assintomáticos corresponderam a 7,7\% e 3,6\%, respectivamente. As formas crônicas foram também predominantes na presente série, entretanto, deve-se observar a alta taxa de etilismo encontrada na população de Manaus (68,4\% para hepatite B e $71,4 \%$ para hepatite C), favorecendo a ocorrência de cirrose hepática. Nos Estados Unidos, $75 \%$ a $85 \%$ dos indivíduos infectados pelo VHC evoluem para formas crônicas; dentre estes, mais de $20 \%$ desenvolvem cirrose hepática ${ }^{(14)}$. Na Holanda, no período de 2002 a 2005 , observou-se $16 \%$ de casos agudos de hepatite B e $80 \%$ de casos crônicos ${ }^{(9)}$. Vale ressaltar que a prevalência de formas crônicas nesta casuística pode não corresponder à prevalência real na população, devido ao fato de que estes casos foram diagnosticados no HSPE e notificados já como formas crônicas. Tal fenômeno decorre também do processo de notificação que utiliza principalmente o laboratório como fonte de informação para a investigação de DNC.

A análise dos dados apresentados mostra que a notificação das hepatites ainda é bastante incompleta, haja vista a grande quantidade de dados epidemiológicos ignorados. Torna-se possível identificar lacunas na coleta de informações epidemiológicas que deveriam ser pesquisadas pelo médico assistente durante a anamnese, assim como falhas do próprio processo de investigação epidemiológica, traduzido pela grande proporção de dados de evolução ignorados. Observase ainda em nosso meio, cobertura vacinal inadequada, fato que provavelmente decorre da faixa etária da amostra, já que a vacina contra hepatite B foi introduzida a partir de 1990 para politransfundidos e renais crônicos e apenas em 1998 foi incluída no calendário básico ${ }^{(5)}$. Estudo realizado em Taiwan mostrou que a prevalência de HBsAg entre universitários diminuiu significativamente após a introdução da vacina contra hepatite B em $1984^{(6)}$, revelando a importância da vacina no perfil epidemiológico desta infecção. 
Dessa forma, conclui-se que a vigilância epidemiológica constitui ferramenta imprescindível para a determinação do risco de infecção e perfil dos pacientes infectados, possibilitando a implementação de medidas de prevenção e controle das hepatites virais. Urge haver melhor coleta de informações epidemiológicas para aprimoramento do processo de investigação e notificação.

Cruz CRB, Shirassu MM, Martins WP. Comparison between hepatitis B and C epidemiological profiles at a public institution in São Paulo, Brazil. Arq Gastroenterol. 2009;46(3):225-9.

ABSTRACT - Context - Viral hepatitis is a disease of compulsory notification and is very important due to its high prevalence. Objective - To analyze the epidemiological profiles of hepatitis B and C, reported through surveillance at the "Hospital do Servidor Público Estadual", São Paulo, SP, Brazil, from January 2004 to April 2007. Methods and Results - The study revealed 112 confirmed cases of hepatitis B and 651 of hepatitis C. Male gender predominated among hepatitis $\mathrm{B}$ while female gender predominated among hepatitis $\mathrm{C}$, statistically significant. Hepatitis $\mathrm{B}$ occurred mainly among patients from 30 to 39 years old $(P<0.01)$, and hepatitis $\mathrm{C}$ was more frequent among patients from 40 to 49 years old $(P=0.49)$. Asthenia was the most frequent symptom. The main risk factors were: sexually transmitted diseases (hepatitis $\mathrm{B} ; P<0.01$ ), previous surgery and blood transfusion (hepatitis $\mathrm{C} ; P<0,01$ ). Cases were mostly confirmed through laboratory tests. Chronic hepatitis occurred in $54.5 \%$ and $81.7 \%$ cases of hepatitis B and C, respectively $(P<0,01)$. Virus B was mostly transmitted by sexual contact $(P<0,01)$; virus $\mathrm{C}$ was transmitted mainly by blood transfusion $(P<0,01)$. Conclusions - The findings were similar to national and international data available. Epidemiologic surveillance plays an important role in determining the viral hepatitis profile in order to provide adequate screening and prevention.

HEADINGS - Hepatitis B, epidemiology. Hepatitis C, epidemiology. Epidemiologic surveillance. Disease notification.

\section{REFERÊNCIAS}

1. Alexander J, Kowdley KV. Epidemiology of hepatitis B--clinical implications. MedGenMed. 2006;8:13.

2. Alter MJ. Epidemiology of hepatitis C virus infection. World J Gastroenterol. 2007;13:2436-41.

3. Araújo A. Hepatites B e C em Manaus: perfil clínico-epidemiológico e distribuição espacial de casos conhecidos desde 1997 a 2001 [dissertação]. Manaus: Fundação Oswaldo Cruz. Escola Nacional de Saúde Pública/Universidade Federal do Amazonas; 2004

4. Brasil. Secretaria de Vigilância em Saúde. Guia de vigilância epidemiológica. 6 . ed. Brasília: Ministério da Saúde; 2005.

5. Centro de Vigilância Epidemiológica do Estado de São Paulo (CVE). Hepatites virais B e C. 2007; http://www.cve.saude.sp.gov.br/htm/hepa_home.html:

6. Chen CC, Yen CH, Wu WY, Hu SW, Chen SC, Bell WR, Lee MC. Epidemiology of hepatitis B virus infection among young adults in Taiwan, China after public vaccination program. Chin Med J (Engl). 2007;120:1155-8.

7. Ferreira C, Silveira T. Hepatites virais: aspectos da epidemiologia e da prevenção. Rev Bras Epidemiol. 2004;7:473-87.

8. Gish RG, Gadano AC. Chronic hepatitis B: current epidemiology in the Americas and implications for management. J Viral Hepat. 2006;13:787-98.

9. Koedijk FD, op de Coul EL, Boot HJ, van de Laar MJ. [Hepatitis B surveillance in the Netherlands, 2002-2005: acute infection is mainly via sexual contact while chronic infection is via vertical transmission through mothers from endemic regions]. Ned Tijdschr Geneeskd. 2007;151:2389-94.
10. Meara M, Barry J, L M. Epidemiology of hepatitis C infection, ERHA/HSE Eastern region. Ir Med J. 2007;100:365-6.

11. Niederau C, Kapagiannidis C. [Epidemiology of hepatitis C in Germany]. Med Klin (Munich). 2006;101:448-57.

12. Peixoto MF, Mattos AA, Remiao JO, Alexandre CO, Lemos SK, Azevedo AM Vertical transmission of hepatitis $\mathrm{C}$ virus in a hospital in southern Brazil. Arq Gastroenterol. 2004;41:84-7.

13. Rustgi VK. The epidemiology of hepatitis $\mathrm{C}$ infection in the United States. J Gastroenterol. 2007;42:513-21.

14. Silva GF, Nishimura NF, Coelho KI, Soares EC. Grading and staging chronic hepatitis $\mathrm{C}$ and its relation to genotypes and epidemiological factors in Brazilian blood donors. Braz J Infect Dis. 2005;9:142-9.

15. Tanaka J. Hepatitis B epidemiology in Latin America. Vaccine. 2000;18(Suppl 1):s17-s9.

16. Tanno H, Fay O. [Viral hepatitis in Latin America]. Acta Gastroenterol Latinoam. 2005;35:169-82.

17. Tanwandee T, Piratvisuth T, Phornphutkul K, Mairiang P, Permpikul P, Poovorawan Y. Risk factors of hepatitis $\mathrm{C}$ virus infection in blood donors in Thailand: a multicenter case-control study. J Med Assoc Thai. 2006;89(Suppl 5):s79-s83.

18. Veronesi L, Verrotti Di Pianella C, Benassi L, Benaglia G, Affanni P, Tanzi ML. Mother to child transmission of hepatitis $\mathrm{C}$ virus in a province of northern Italy J Prev Med Hyg. 2007;48:47-9. 